Journal of Animal and Veterinary Advances 18 (6): 187-194, 2019

ISSN: 1680-5593

(C) Medwell Journals, 2019

\title{
Epidemiology and Genetic Variants within Echinococcus granulosus Identified based on ITS-1 Ribosomal DNA in North-West Egypt
}

\author{
${ }^{1}$ Safaa Mohamed Barghash and ${ }^{2}$ Ahmed Mohamed Darwish \\ ${ }^{1}$ Desert Research Center, Parasitology Unit, Department of Animal Health, \\ PO Box 11753, Cairo, Egypt \\ ${ }^{2}$ National Research Center, Department of Cell Biology, \\ Genetic Engineering and Biotechnology Division, Giza, Egypt
}

\begin{abstract}
Echinococcus granulosus, the etiologic agent of Cystic Echinococcosis (CE) is one of the most important zoonotic helminths with worldwide distribution in humans and animals. The current study aimed to survey the population genetic pattern of CE isolated from humans and slaughtered animals in North-West Egypt by sequencing of ITS-1 ribosomal DNA. A total of 4047 animals (1512 camels, 1575 cattle, 192 donkeys, 867 sheep, 703 goats, 98 buffaloes) and 100 suspected cases in humans were surveyed, for their fertility and viability. It was collected from different abattoirs and hospitals in North-West Egypt and identified by using nested-PCR-ITS1 of rDNA. The isolates amplify $800 \mathrm{bp}$ were sequenced and BLASTn. Nucleic acid sequence maximum likelihood method was used to create the consequence tree and subsequent phylogeny to compare these sequences with globally and retrieved reference strains of Echinococcus granulosus from GenBank. Results revealed that 155 hydatid cysts were found fertile and viable and consequently subjected to nPCR-ITS1 of rDNA. Molecularly, they identified as 99 (63.9\%) were G6 strain whereas 56 (36.1\%) were as G1 strain. The similarity between submitted isolates and preserved from the same species deposited in GenBank ranged from 98.3-96.9\%. It was more similar to Australian and Indian sheep than Iranian isolates. We concluded that the sheep G1 and camel G6 genotypes of Echinococcus granulosus are prevalent with the possibility of transmission between livestock animals and humans are likely to be exposed to G1 strain in North-West Egypt.
\end{abstract}

Key words: Echinococcus granulosus, G1, G6, nPCR-ITS1, Gene sequencing, Phylogenetic tree, Egypt

\section{INTRODUCTION}

Cystic Echinococcosis (CE) caused by Echinococcus granulosus is an emerging and re-emerging disease in several countries and regions, even in places where it was previously at low levels, especially in developing countries, leading to a public health problem of increasing concern (Sharafi et al., 2014). Canids, particularly dogs are important in zoonotic transmission because of their close relationships with humans and ruminants. Intermediate hosts are initially asymptomatic; however, larval infection is characterized by the long-term growth of hydatid cysts in the intermediate host's tissues (Seres et al., 2009)

Hydatid cysts act like tumors can disrupt the function of vital organs where they are found and can lead to illness and death (Nur et al., 2017). Large or multiple cysts may cause irreversible damage to organs. The rupture or puncture of the cyst into the peritoneal cavity leads to secondary echinococcosis which can seed multiple organs with larvae or cause anaphylactic reactions (Khachatryan, 2017). Most livestock is slaughtered before the cysts become large enough to cause clinical signs but if dogs feed their entrails, it perpetuates the cycle. Animals that live long enough such as horses, may become ill (Sako et al., 2011).

The extensive variation in E. granulosus may affect life cycle, host specificity, development rate, sensitivity to chemotherapeutic agents plus pathology and consequently for design and development of vaccines against E. granulosus (Sharbatkhori et al., 2010). Recently, PCR and DNA sequence analyses have been widely applied to phylogenetic relationships and determining the interspecific and intraspecific genetic variations of the parasite in various parts of the world. It mainly based on mtDNA, NAD1, COX1-genes and rarely by nuclear DNA markers (PCR-RFLP of ITS1) (Amer et al., 2015; Mohaghegh et al., 2018; Torgerson and Budke, 2003).

To date, E. granulosus complex consists of four species comprise ten defined strains (genotype G1-11) with worldwide distribution in human and animal

Corresponding Author: Safaa Mohamed Barghash, Desert Research Center, Parasitology Unit, Department of Animal Health, P.O. Box 11753, Cairo, Egypt Fax: +20 226357858 
(Sharbatkhori et al., 2010). Except for G4 genotype, all other strains have been found to infect humans (Moro and Schantz, 2009). Many countries in North Africa and the Middle East are highly endemic to E. granulosus with many studies focusing on CE genotypes, especially in Iran (Berinji et al., 2019; Craig et al., 2005; Lahmar et al., 2004).The importance of small ruminants, particularly sheep as an intermediate host in these regions has been demonstrated (Banda et al, 2012; Gholami et al, 2012; Khan et al., 2001). Human cases are regularly observed and widespread recovery of adult worms has been reported from dogs and often camel is an important reservoir for human infection (El Sayed et al., 2017; Khan et al., 2001).

In Egypt, thousands of camels and sheep are imported annually from East African Countries and Australia, respectively. A few comprehensive studies carried out thus far to map and describe CE in both the intermediate and final hosts and others have indicated an increased prevalence rate in animals and humans in the last few years (Abdel Aaty et al., 2012; Hosseini et al, 2012). Some of them are still poorly characterized and further research is needed to determine in higher detail their hosts and geographic ranges and whether their genetic characteristics are conserved between different regions (Ahmadi and Dalimi, 2006). Therefore, the potential role of hydatid cysts in slaughtered animals for CE is epidemiologically important in addition to their molecular characterization. We hope that this finding might be considered as a complementary part of the puzzle on determining the gene map of the parasite in Egypt.

\section{MATERIALS AND METHODS}

Study area: The current study was carried out in Matrouh governorate, the second largest province in terms of area in Egypt. It represented the North-West Coastal zone (NWC) from West-Delta to El-Salloum in the West. Animal husbandry and tourism are the main sources of income. Millions of vacationers go to it each year in Summer consuming thousands of local animals and others are taken from other provinces and neighboring countries for slaughter.

Sample collection and microscopical examination: A total of 4049 hydatid cysts (one from each animal) were collected from the livers, lungs and gastrointestinal tract of 1512 camels, 1575 cattle, 192 donkeys, 867 sheep, 703 goats and 98 buffaloes from January, 2014 to March, 2018. Besides, 100 liver samples from humans suspected to have infections with CE collected from hospitals. Cysts were processed separately and protoscolices were aspirated and washed several times with normal saline until the supernatant looked clear and the sediment preserved in $70 \%$ ethanol. The viable cysts were washed to remove ethanol, examined microscopically and then stored at $-20^{\circ} \mathrm{C}$ until used for PCR amplification.

DNA extraction and PCR-ITS1 amplification: Genomic DNAs of 155 fertile and viable samples were extracted from protoscolices using commercially available DNeasy Blood and Tissue Kit (QIAGEN Inc. Germany), following the manufacturer's instructions. DNAs were amplified by nPCR of ITS1-rDNA produced 800 bp as described previously by (Rahimi et al., 2007). The PCR was performed in two steps by using forward and reverse specific primers: EgF/EgR to detect $1000 \mathrm{bp}$ and EGF1/EGR2 to detect 1800 bp (Invitrogen, Life Technologies). The marker used with $100 \mathrm{bp}$ molecular weight (Sigma, USA).

ITS-1 ribosomal DNA sequencing and phylogenetic analysis: Six specific bands at 800 bp generated from 6 different species isolates after performing nPCR were cut from the gel and purified using Bio Basic Purification Kit (Canada), according to the manufacturer's instructions, then sequenced in a forward direction by Macrogen (Korea). The sequences were compared to the sequences deposited in GenBank using the BLAST system (http://www.ncbi.nlm.nih.gov/) and BioEdit Software. Phylogenetic analysis carried out ITS-gene isolated from Egyptian isolates as well as reference sequences of identified E. granulosus genotypes (Table 1). Nucleic acid sequence maximum likelihood method, Version 3.3 of BioEdit Software was used to create the consequence tree.

\section{RESULTS AND DISCUSSION}

Prevalence of cyst fertility and viability: Out of the 5047 humans and animals examined, 650 (12.8\%) were found harboring hydatid cysts. The most species affected were sheep (27.2\%), goats (23.9\%), camels (13.7\%), donkeys (8.85\%), cattle with a low percentage $(1.3 \%)$ and human $(1.00 \%)$ while buffaloes were free from infection. Whilst the lung was the main target organ involved in large animals, the Gastrointestinal Tract (GIT) and the liver were the organs target in sheep, goats and donkeys. Moreover, their cysts were larger in size than those of small ruminants. Of the cysts found, 291 (44.8\%) were considered fertile with the presence of protoscolices. Camels possessed the highest cyst fertility rate $(70.1 \%)$, followed by goats $(37.5 \%)$ and sheep (33.1\%). Out of the fertile cysts observed, only 155 (53.3\%) were found viable whereas 136 (46.7\%) were dead (Table 1). 
J. Anim. Vet. Adv., 18 (6): 187-194, 2019

Table 1: Prevalence and status of hydatid cysts of human and different slaughtered animals

\begin{tabular}{lllllllll} 
Animals & Camel & Sheep & Goat & Donkey & Buffalo & Cattle & Human & Total \\
\hline No. examined & 1512 & 867 & 703 & 192 & 98 & 1575 & 100 & 5047 \\
Hydatid Cyst (HC) (\%) & $207(13.7)$ & $236(27.2)$ & $168(23.9)$ & $17(8.85)$ & $0.00(0.00)$ & $21(1.3)$ & $1(1.00)$ & $650(12.8)$ \\
Infertile cyst (\%) & $62(29.95)$ & $158(66.95)$ & $105(62.5)$ & $14(82.4)$ & $0.00(0.00)$ & $20(95.2)$ & $0.00(0.00)$ & $359(55.2)$ \\
Fertile cysts (\%) & $145(70.1)$ & $78(33.1)$ & $63(37.5)$ & $3(17.6)$ & $0.00(0.00)$ & $1(4.8)$ & $1.00(1.00)$ & $291(44.8)$ \\
Viable (\%) & $111(76.6)$ & $21(26.9)$ & $19(30.2)$ & $2(66.7)$ & 0.00 & $1(100)$ & $1(100)$ & $155(53.3)$ \\
Non-viable (\%) & $34(23.5)$ & $57(73.1)$ & $44(69.8)$ & $1(33.3)$ & $0.00(0.00)$ & $0.00(0.00)$ & $0.00(0.00)$ & $136(46.7)$ \\
\hline
\end{tabular}

HC: Hydatid Cyst, Infertile (sterile or calcified/HC), Fertile (have protoscolices/HC), Viable (live protoscolices/fertile) and non-viable (dead/fertile)

Table 2: Number of isolates and their sizes of the DNA fragments (bp) after PCR amplification with specific primers and after nPCR

\begin{tabular}{lcclc}
\hline Original host & Viable cysts & EGF1/EGR2 (bp) & EgF/EgR (bp) & After nPCR (bp) \\
\hline Camel & 111 & 1800,800 & 1000 & 800 \\
Donkey & 2 & 1800 & - & 800 \\
Sheep & 21 & - & 1000,800 & 800 \\
Cattle & 1 & 1000 & - & 800 \\
Goat & 19 & - & $1000,800,600$ & 800 \\
Human & 1 & 1000 & - & 800 \\
\hline
\end{tabular}

(a)

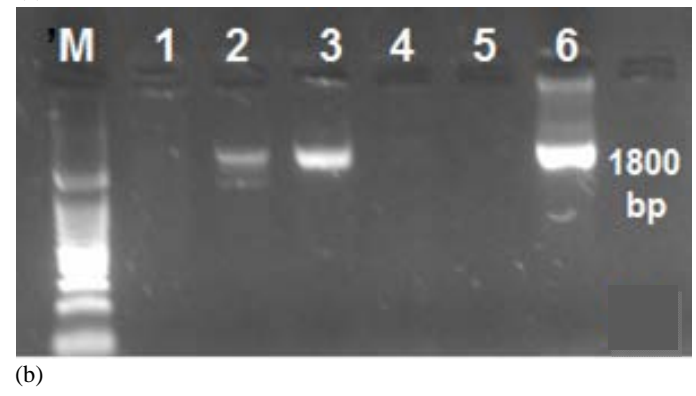

(b)

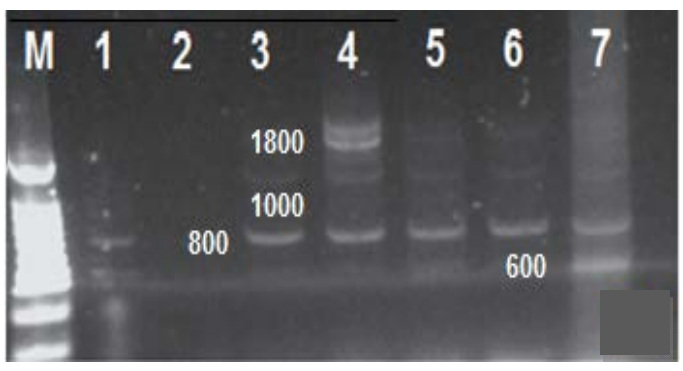

Fig. 1(a-b): PCR of rDNA-ITS1 showed generated fragments on agarose gel from DNAs of hydatid cysts isolated from human and animals by using (a) EGF1/EGR2 and (b) EgF/EgR specific primers

ITS1-PCR amplification: ITS1-PCR was successfully used to identify E. granulosus DNAs isolated from hydatid cysts recovered from 155 different animals and human isolates without PCR inhibitors (Fig.1a, b). Two banding patterns of $E$. granulosus were obtained with the individual outer and inner specific primers as shown in Table 2. Bands of 1800 bp confirmed the existence and dominance of G6 (camel strain) in the import and majority of local camel samples plus donkeys isolates. The other genotype G1 produced bands of 1000 bp (sheep strain) found in other studied isolates. It was identified as 99 (63.9\%) G6 strain whereas 56 (36.1\%) was G1 strain.
G1 primers could generate other bands at 600 in goats isolates. After nPCR, the different isolates gave similar patterns at $800 \mathrm{bp}$, in spite of diverse host and geographical origins.

Gene sequencing and single nucleotide polymorphisms in ITS-1: The six samples representative of different species were introduced to GenBank with coded names CT, N, C1, G, CO and M isolated from sheep, human, camel, goat, cow and donkey, respectively. The ITS1 gene amplicons produced by nPCR was 800 bp long and the consensus lengths of ITS1 gene sequence was $724 \mathrm{bp}$. E. granulosus of these isolates shared in the following SNPs: T, A and $G$ in different positions (Table 3). Nucleotide sequencing of the submitted isolates revealed the occurrence of nucleotide mutations generating a single nucleotide polymorphism positions comparing to reference sequences at 26, 467 and 473 nucleotide where a Thymine (T) replaced a Guanine $(\mathrm{G})$ and at positions of 212, 213 and 618 nucleotides, Adenine (A) substituted a Guanine (G). At SNPs positions 73, 135, 410 and 572 nucleotides, a Guanine $(\mathrm{G})$ replaced Adenine (A). In contrast, at the position 522 nucleotide, Adenine (A) substituted a Guanine (G). Also, 2 nucleotide substitutions (both Adenine to Cytosine) at positions 316 in human and goat isolates and 3 nucleotide substitutions (all GTG to TGA) at positions 233, 234 and 235.

Phylogenetic analysis: The ratio of similarity between applied samples and E. granulosus isolated from the same animals and deposited in GenBank is ranged from 98.3-96.9. The highest similarity was 98.3 between the human isolate and Australian sheep. All submitted samples similar to Australian or Indian sheep more than Iranian cattle, goat, sheep and camel isolates (Table 4). From the evolutionary relationship, it is clear that E. granulosus is very close together and distant to other Echinococcus species (Oligarthrus, Ortelippy, 
J. Anim. Vet. Adv., 18 (6): 187-194, 2019

Table 3: Position of SNPs in ITS1 gene of applied samples compared with Echinococcus granulosus deposited in GenBank

\begin{tabular}{|c|c|c|c|c|c|c|}
\hline & \multicolumn{6}{|c|}{ Egyptian isolates } \\
\hline & Sheep & Human & Camel & Goat & Cow & Donkey \\
\hline Nucleotide position & CT & $\mathrm{N}$ & C1 & G & Co & $\mathrm{M}$ \\
\hline $26,233,467,473$ & $\mathrm{~T}$ & $\bar{T}$ & $\mathrm{~T}$ & $\mathrm{~T}$ & $\mathrm{~T}$ & $\mathrm{~T}$ \\
\hline 212, 213, 235618 & A & A & A & A & A & A \\
\hline $73,135,234,410,572$ & G & G & G & G & G & G \\
\hline 316 & - & A & - & A & - & - \\
\hline 522 & G & - & G & - & G & G \\
\hline 719 & - & A & - & - & A & - \\
\hline
\end{tabular}

Table 4: Ratio of similarity between the submitted isolates and the most related reference Echinococcus granulosus deposited in GenBank

\begin{tabular}{|c|c|c|c|c|c|c|c|}
\hline & & Sheep & Human & Camel & Goat & Cow & $\overline{\text { Donkey }}$ \\
\hline Identity (Reference) & Accession No. & CT & $\mathrm{N}$ & CI & G & $\mathrm{CO}$ & M \\
\hline Sheep-Iran (Arbabi et al., 2017) & KJ363922 & 97.0 & 97.0 & 97.2 & 97.2 & 97.0 & $\overline{97.2}$ \\
\hline Goat-Iran (Arbabi et al., 2017) & KJ363927 & 97.0 & 97.0 & 97.2 & 97.2 & 97.0 & 97.2 \\
\hline Camel-Iraq (Hansh and Awad, 2016) & LT547814 & 97.5 & 97.3 & 97.5 & 97.2 & 97.6 & 97.6 \\
\hline Sheep-Australia (Van Herwerden et al., 2000) & AF132700 & 98.0 & 98.3 & 98.0 & 97.7 & 97.9 & 97.7 \\
\hline Sheep-India & KY129666 & 97.9 & 97.9 & 98.0 & 98.0 & 98.0 & 98.2 \\
\hline Cattle-India (Bhattacharya et al., 2008) & AY969044 & 97.0 & 97.2 & 96.9 & 97.0 & 97.0 & 96.9 \\
\hline
\end{tabular}

Multilocularis, Equinus, Canadensis and Felidis). Echinococcus isolates in the current study were clustered together in one clade closely related to $E$. granulosus of Australian sheep. It is also close to E. granulosus isolated from Iranian sheep and goat. In contrast, it is distant to E. granulosus isolated from Iranian camel and cattle as demonstrated in Fig. 2a, b.

The current study was one of the most comprehensive studies in domestic animals performed in Egypt involving cases originating from all geographic regions, except for the New-Valley and Sinai-Peninsula. It came to complete our study on CE in Egypt which started with a big survey in humans and slaughtered animals in Delta, Great Cairo and Upper Egypt (Barghash et al., 2017). In the present study, we discuss the epidemiological survey concerning genotype $E$. granulosus circulating in the study area and to define the role of intermediate hosts. We performed a greater number of animals but the high frequency of infection in small ruminants and camels from abattoirs must be of concern about the real situation of this zoonosis. Furthermore, it was found that offal is consumed by the community rather given to dogs around slaughterhouses, especially in desert areas. This may increase the chance of environmental contamination, whereby dogs can easily acquire the infection and then continuously discharge eggs of Echinococcus parasites as reported by (Boru et al., 2013).

In the present study, it is clear that imported camels, then sheep and goats are by far the most frequently infected intermediate host species in agreement with (Barghash et al., 2017). Local camels are found in arid regions of Egypt and commonly infected with E. granulosus, possessing a high cyst fertility rate, followed by goats and sheep. This high prevalence could be attributed to high numbers of camels and dog populations coupled with a high number of home slaughters during ceremonies which in some cases are not inspected by the veterinary department staff in agrees with Gholami et al. (2012). This finding of viable camel's $\mathrm{CE}$ is rather low when compared to the results reported of other countries like Morocco (80\%), Algeria (56.5\%), Sudan (45.4\%), Tunisia (34.5\%), Libya (31.9\%) and Iran (25.7-59.3\%) (Banda et al., 2012; Berinji et al., 2019; Craig et al., 2005; Gholami et al., 2012; Lahmar et al., 2004; Moro and Schantz, 2009; Rokni, 2009). In contrast, the present results are roughly higher than reported previously by (Arbabi et al., 2017; Rahimi et al., 2007) that ranged from 3.5-5\% for camels, sheep (3.9-14.1\%) whereas goats were $4.5-13 \%$, respectively.

Concerning other intermediate hosts, cattle were found infrequently affected that a great proportion of their cysts are sterile and are typical of a bad adaptation of the parasite to its host which would be abnormal and play no significant role, like buffaloes in the epidemiology of E. granulosus in Egypt as reported by (El Sayed et al., 2017). Whereas sheep exhibited a slightly greater proportion of fertile cysts but lower than this of dromedaries and there are many infertile cysts. These results are in contrast to that recorded in ruminants in Iran where sheep have (5.1-74.4\%), goats (2-20\%), cattle (3.5-38.3\%) and buffalo (11.9-70\%) (Dalimi et al., 2002). Furthermore, lungs in large animals and livers in other hosts were the most affected organs compared to GIT. This is explained as the lungs and livers are the first capillary beds encountered by migrating Echinococcus oncospheres via. the portal vein route 

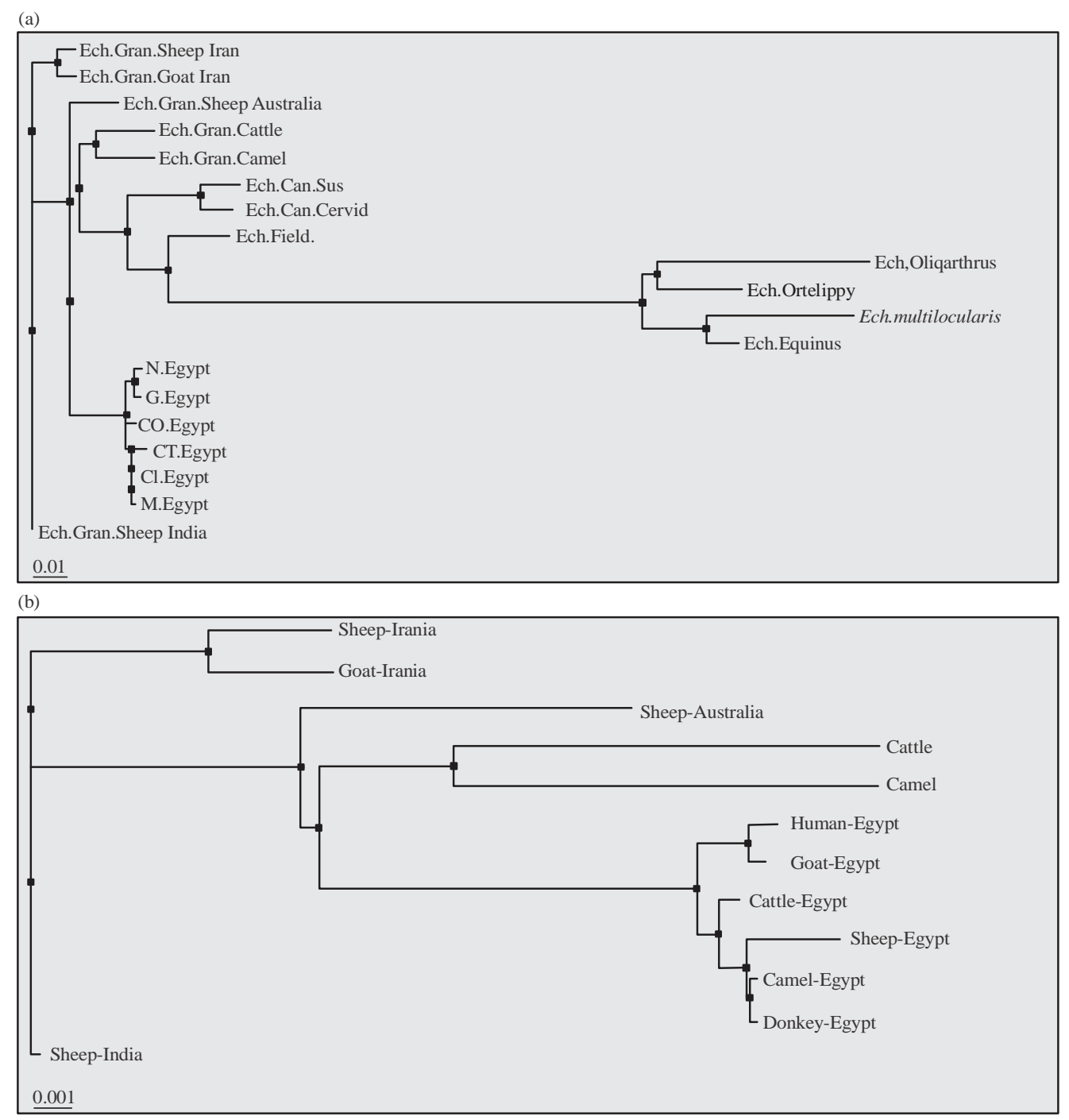

Fig. 2(a-b): Phylogenetic trees generated based on rDNA-ITS1 results for the applied Echinococcus granulosus isolates (N, G, Co, Ct, C1 and M) and reference Echinococcus species/strains with the two primers used, showing similarity between them

before any other peripheral organs are in agreement with (Abdel Aaty et al., 2012; Ahmadi and Dalimi, 2006; Hosseini et al., 2012).

Notably, E. granulosusis maintained in two dominate and distinct cycles, a mainly desert cycle between dogs and camel, followed by domestic cycle which involves livestock and dogs as the intermediate and definitive host, respectively. However, most livestock is slaughtered before the cysts become large enough to cause clinical signs in coinciding (Sako et al., 2011; Barghash et al., 2017). The geographic distribution differs by region depending on the presence of large numbers of nomadic or semi-nomadic sheep and goat flocks from NWC. Local and imported camels also represent the intermediate hosts of the parasite and their close contact with the final host, the dog. Besides, the factor governing the prevalence of
CE in a given locality may be associated with prevailing specific social-cultural, environmental conditions and the dynamics of transmission between the dog and its intermediate host in agreement with (Abdel Aaty et al., 2012).

In Egypt, except for a few molecular studies have recorded only the presence of the camel strain (G6), equine strain (G4) and buffalo strain (G5) in definite regions and advised to use gene sequencing of ITS1-PCR (Craig et al., 2005). Many studies have shown clearly that we face a predominant sheep strain (G1) with a high percentage (Abd El Baki et al., 2009; Aboelhadid et al., 2013; Khalifa et al., 2014; Taha, 2012). The current study supported the prevalent and dominance of G1 and G6 E. granulosus in most parts of Egypt, especially in rural areas where slaughter is practiced on farms. Whereas G1 
and G6 life cycles patterns overlap with intermediate hosts where camels occur together with small ruminants and dogs. It also confirmed that G1 genotype of E. granulosus is dominant in small ruminants from different regions in Egypt and humans are likely to be exposed to the camel strain G6 as well as the sheep strain G1 through contact with dogs. It is in concordance with (Aboelhadid et al., 2013) who detected G1 genotype in 83.3\% Egyptian human isolates of HC by PCR without significant difference between types of human isolates (pulmonary, hepatic or multi-organ). The outcome is in line with several previous studies recording that G1 and G6 were identified most frequently in Egypt, Iran, Tunisia and Ethiopia (Moro and Schantz, 2009).Furthermore, donkeys may have the same genotypic characters of CE isolated from camels in agreement with (Taha, 2012).

Take into consideration that a few studies used nPCR-ITS1 of rDNA on contrary to NAD1, COX1-mDNA, consequently, a decrease in the number of the reference E. granulosus strains deposited in GenBank (Amer et al., 2015, Khalifa et al., 2014). In the current study, the ITS sequences obtained from human and animals isolates were between $96.9-98.3 \%$ homologous to sequences of $E$. granulosus strains previously detected in Iran, India and Australia. It revealed the occurrence of nucleotide mutations generating SNPs sequencing of the Egyptian isolates at 16 nucleotide positions and demonstrated that it is positioned in the same clade as other E. granulosus isolates based on the phylogenetic analysis. This variation may play an important role in not only transmission patterns but also pathogenicity, fertility and growth rate of hydatid cysts as reported by (Sharma et al., 2013). It agrees with (Amer et al., 2015) who provided evidence that E. granulosus in Egypt is not a monophyletic taxon and strains within this species fall into groups which might merit recognition as separate species when he examined 93 isolates from sheep and camels using three-nucleotide datasets (COX1, NAD1 and rRNA). On the other hand, the highest similarity and close relationship between the reference Australian sheep and the human isolate may indicate to a role in spreading CE in Egypt, especially, thousands of Australian sheep are imported and slaughtered in celebrations and festivals. This suggestion needs further evaluation in the next studies, especially, the human case thought to be sourced from Australian sheep used to eat it.

\section{CONCLUSION}

G1 and G6 strains are prevalent and represent important aspects of the epidemiology of E. granulosus in North-West Egypt. G1 can potentially act as a risk factor for human echinococcosis.

\section{ABBREVIATIONS}

ITS1 : Ribosomal Internal Transcribed Spacer 1 nad1 : Mitochondrial NADH dehydrogenase subunit 1 cox1 : Mitochondrial cytochrome c oxidase subunit rRNA : Ribosomal RNA

\section{AKNOWLEDGEMENTS}

The researchers gratefully acknowledge the financial support from the Desert Research Center (DRC) and the National Research Center (NRC). We would also like to thank the workers in slaughtered houses for their help during this study.

\section{REFERENCES}

Abd El Baki, M., A. El Missiry, H. Abd El Aaty, A. Mohamad and H. Aminou, 2009. Detection of G1 genotype of human cystic echinococcosis in Egypt. J. Egypt. Soc. Parasitol., 39: 711-721.

Abdel Aaty, H.E., D.M. Abdel-Hameed, Y.H. Alam-Eldin, S.F. El-Shennawy, H.A. Aminou, S.S. Makled and S.K. Darweesh, 2012. Molecular genotyping of Echinococcus granulosus in animal and human isolates from Egypt. Acta Tropica, 121: $125-128$.

Aboelhadid, S.M., K.M. El-Dakhly, T. Yanai, H. Fukushi and K.M. Hassanin, 2013. Molecular characterization of Echinococcus granulosus in Egyptian donkeys. Vet. Parasitol., 193: 292-296.

Ahmadi, N. and A. Dalimi, 2006. Characterization of Echinococcus granulosus isolates from human, sheep and camel in Iran. Infect. Genet. Evol., 6: 85-90.

Amer, S., I.B. Helal, E. Kamau, Y. Feng and L. Xiao, 2015. Molecular Characterization of Echinococcus granulosus Sensu Lato from farm animals in Egypt. PloS One, Vol. 10. 10.1371/journal.pone. 0118509

Arbabi, M., M. Pirestani, M. Delavari, H. Hooshyar, A. Abdoli and S. Sarvi, 2017. Molecular and morphological characterizations of Echinococcus granulosus from human and animal isolates in Kashan, Markazi Province, Iran. Iran. J. Parasitol., 12: 177-187.

Banda, F., K.S. Nalubamba, J.B. Muma, M. Munyeme and H. Mweemba Munang'andu, 2012. A cross-sectional study investigating cystic hydatidosis in slaughtered cattle of Western Province in Zambia. ISRN. Parasitol., Vol. 2013, 10.5402/2013/468163

Barghash, S.M., R.A. El Sayed, N.M. El-Alfy, B.M. Abou-Elnour and A.M. El-Kattan, 2017. Prevalence and molecular identification of Echinococcus granulosus in humans and slaughtered animals in Egypt. EJBPS. Eur. J. Biomed. Pharm. Sci., 4: 34-42.

Berenji, F., S.A. Shamsian, M.N. Daloee, S.H.F. Masoom and E. Moghaddas, 2019. Genotyping of Echinococcus granulosus isolates from human in Khorasan Province, North-Eastern Iran. Iran. J. Parasitol., 14: 52-58. 
Bhattacharya, D., A.K. Bera, B.C. Bera, D. Pan and S.K. Das, 2008. Molecular appraisal of Indian animal isolates of Echinococcus granulosus. Indian J. Med. Res., 127: 383-387.

Boru, B.G., Y.H. Tolossa, G. Tilahun and H. Ashenafi, 2013. Study on prevalence of hydatidosis and cyst characterization in camels (Camelus dromedaries) slaughtered at Akaki abattoir, Ethiopia. J. Vet. Med. Anim. Health, 5: 329-333.

Craig, P., A. Mastin, V.F. Kesteren and B. Boufana, 2005. Echinococcus granulosus: Epidemiology and state-of-the-art of diagnostics in animals. Vet. Parasitol., 213: 132-148.

Dalimi, A., G. Motamedi, M. Hosseini, B. Mohammadian, H. Malaki, Z. Ghamari and F.G. Far, 2002. Echinococcosis/hydatidosis in Western Iran. Vet. Parasitol., 105: 161-171.

El Sayed, R.E., S.M. Barghash, N.M. El-Alfy, B.M. Abou-Elnour and S.M. Sadek, 2017. Physiological, immunological and histopathological comparison of Echinococcus granulosus (G6) camel strain by different viability status using secondary cyst development in rat. Int. J. Adv. Res., 5: 2119-2131.

Fasihi Harandi, M., R.P. Hobbs, P.J. Adams, I. Mobedi, U.M. Morgan-Ryan and R.C.A. Thompson, 2002. Molecular and morphological characterization of Echinococcus granulosus of human and animal origin in Iran. Parasitology, 125: 367-373.

Gholami, S., M. Sosari, M. Fakhar, M. Sharif, A. Daryani, M.B. Hashemi and M. Vahadi, 2012. Molecular characterization of Echinococcus granulosus from Hydatid Cysts isolated from human and animals in Golestan Province, North of Iran. Iran. J. Parasitol., 7: 8-16.

Hansh, W.J. and A.H.H. Awad, 2016. Genotyping study of hydatid cyst by sequences of ITS1-rDNA in Thi-Qar-Southern of Iraq. Int. J. Curr. Microbiol. App. Sci., 5: 350-361.

Hosseini, S.H., A.A. Pour and P. Shayan, 2012. Morphological characteristics of Echinococcus granulosus derived from Buffalo in Iran. Parasitol., 139: 103-109.

Khachatryan, A.S., 2017. Analysis of lethality in Echinococcal disease. Korean J. Parasitol., 55: 549-553.

Khalifa, N.O., H.F. Khater, H.A. Fahmy, M.E.I. Radwan and J.S.A. Afify, 2014. Genotyping and phylogenetic analysis of cystic echinococcosis isolated from camels and humans in Egypt. Am. J. Epidemol. Infect. Dis., 2: 74-82.
Khan, A.H., A.A. El-Buni and M.Y. Ali, 2001. Fertility of the cysts of Echinococcus granulosus in domestic herbivores from Benghazi, Libya and the reactivity of antigens produced from them. Ann. Trop. Med. Parasitol., 95: 337-342.

Lahmar, S., H. Debbek, L.H. Zhang, D.P. McManus, A. Souissi, S. Chelly and P.R. Torgerson, 2004. Transmission dynamics of the Echinococcus granulosus sheep-dog strain (G1 genotype) in camels in Tunisia. Vet. Parasitol., 121: 151-156.

Mohaghegh, M.A., H. Yousefi-Darani, M. Azami, M. Ghomashlooyan and N. Hashemi et al., 2018. Analysis of the cox1 gene in Echinococcus granulosus from sheep in northeast Iran using PCR high-resolution melting (qPCR-HRM) curve analysis. Trop. Biomed., 35: 91-99.

Moro, P. and P.M. Schantz, 2009. Echinococcosis: A review. Int. J. Infect. Dis., 13: 125-133.

Muskin, S., D. Hailu and Y. Moti, 2011. Infection rates, cyst fertility and larval viability of hydatid disease in camels (Camelus dromedaries) slaughtered in Kaliti abattoir from Borena, Kereyu and Harar areas of Ethiopia. Glob. Vet., 7: 518-522.

Nur, A., B. Abera and T. Gunse, 2017. The significance (Socio-Economic Impact) and control of Echinococcosis/Hydatidosis: A review. Eur. J. Biol. Sci., 9: 58-66.

Rahimi, H.R., E.B. Kia, S.H. Mirhendi, A. Talebi, M.H. Fasihi, N. Jalali-z and M.B. Rokni, 2007. A new primer pair in its1 region for molecular studies on echinococcus granulosus. Iran. J. Pub. Health, 36: 45-49.

Rokni, M.B., 2009. Echinococcosis/hydatidosis in Iran. Iran. J. Parasitol., 4: 1-16.

Sako, Y., D. Tappe, K. Fukuda, Y. Kobayashi and S. Itoh et al., 2011. Immunochromatographic test with recombinant Em18 antigen for the follow-up study of alveolar echinococcosis. Clin. Vaccine Immunol., 18: 1302-1305.

Seres, S., A. Iovu, M. Junie and V. Cozma, 2009. Prevalence of Echinococcus granulosus Cluj county, Romania, revealed by PCR. Rev. Sci. Parasitologica, 10: 68-71.

Sharafi, S.M., M. Rostami-Nejad, M. Moazeni, M. Yousefi, B. Saneie, A. Hosseini-Safa and H. Yousofi-Darani, 2014. Echinococcus granulosus genotypes in Iran. Gastroenterol. Hepatol. Bed Bench, 7: 82-88.

Sharbatkhori, M., H. Mirhendi, M.F. Harandi, M. Rezaeian and M. Mohebali et al., 2010. Echinococcus granulosus genotypes in livestock of Iran indicating high frequency of G1 genotype in camels. Exp. Parasitol., 124: 373-379. 
Sharma, M., B.A. Fomda, S. Mazta, R. Sehgal, B.B. Singh and N. Malla, 2013. Genetic diversity and population genetic structure analysis of Echinococcus granulosus sensu stricto complex based on mitochondrial DNA signature. PLoS One, Vol. 8, No. 12. 10.1371/journal.pone.0082904

Taha, H.A., 2012. Genetic variations among Echinococcus granulosus isolates in Egypt using RAPD-PCR. Parasitol. Res., 111: 1993-2000.
Torgerson, P.R. and C. Budke, 2003. Echinococcosis: An international public health challenge. Res. Vet. Sci., 74: 191-202.

Van Herwerden, L., R.B. Gasser and D. Blair, 2000. Its ribosomal DNA sequence variants are maintained in different species and strains of Echinococcus. Int. J. Parasitol., 30: 157-169. 